\title{
Comunicação
}

[Communication]

\section{Ocorrência de Spalangia endius Walker, 1839 (Hymenoptera, Pteromalidae) em pupas de Musca domestica L. e Stomoxys calcitrans L. (Diptera, Muscidae) no sul do Rio Grande do Sul}

[Occurrence of Spalangia endius Walker, 1839 (Hymenoptera, Pteromalidae) in pupae of Musca domestica L. and Stomoxys calcitrans L. in Southern Rio Grande do Sul, Brazil]

\author{
R.K. Brandão ${ }^{1}$, M.C. Cárcamo ${ }^{1,3}$, V.A. Costa $^{2}$, P.B. Ribeiro ${ }^{1}$ \\ ${ }^{1}$ Departamento de Microbiologia e Parasitologia - IB-UFPel \\ Caixa Postal 354 \\ 96010-900 - Pelotas, RS \\ ${ }^{2}$ Centro Experimental Central do Instituto Biológico - IB-APTA - Campinas, SP \\ ${ }^{3}$ Bolsista da CAPES
}

\begin{abstract}
Altas densidades populacionais de Musca domestica Linnaeus, 1758 (Diptera, Muscidae) e Stomoxys calcitrans Linnaeus, 1758 (Diptera, Muscidae) em aviários e criações bovinas, associadas à extrema mobilidade desses insetos adultos, indicam a necessidade de um manejo adequado, devido à expansão da urbanização e às doenças promovidas por esses insetos (Kaufman et al., 2001).
\end{abstract}

O díptero, conhecido popularmente como moscados-estábulos, S. calcitrans, inseto hematófago comumente encontrado em criações de animais, ataca bovinos, ovinos, caprinos e equinos, causando queda de 20 a $60 \%$ na produção leiteira. $M$. domestica é uma das espécies de mosca de maior interesse em saúde pública devido a seu caráter sinantrópico, à capacidade de desenvolvimento em vários substratos e ao alto poder reprodutivo (Mariconi et al., 1999). Essas duas espécies de moscas são veiculadoras de bactérias, vírus, protozoários e helmintos (Moraes et al., 2004). Ao transportarem patógenos através do aparelho bucal, fezes e pernas, contaminam alimentos, água e utensílios, causando males ao homem e aos animais.

Apesar do grande número de inimigos naturais de moscas, os programas de controle biológico têm dado ênfase ao uso de parasitoides (Mariconi

Recebido em 23 de outubro de 2010

Aceito em 25 de fevereiro de 2011

*Autor para correspondência (corresponding author)

E-mail: rosikb@ibest.com.br et al., 1999). Os parasitoides são agentes responsáveis pela redução da população de moscas que proliferam em esterco, cadáveres e carcaças de animais. Os himenópteros parasitoides desempenham importante papel na regulação das populações de pragas, pois sempre matam seus hospedeiros. São espécies densodependentes das populações de seus hospedeiros, o que os torna importantes na manutenção do balanço ecológico e diversidade de outros organismos.

O microimenóptero Spalangia endius Walker, 1839 (Hymenoptera, Pteromalidae) desenvolvese como endoparasitoide solitário de pupas de dípteros das famílias Anthomyiidae, Calliphoridae, Muscidae, Sarcophagidae e Tephritidae. Berti Filho et al. (1996), ao revisarem a bibliografia sobre artropodofauna benéfica associada ao esterco de galinhas poedeiras, no Brasil, registraram S. endius como parasitoide de pupas de $M$. domestica, Muscina stabulans (Fallén, 1817) (Diptera, Muscidae), S. calcitrans e Chrysomya putoria (Wiedemann, 1818) (Diptera, Calliphoridae). Mencionaram, ainda, que $S$. endius está entre os agentes com maior capacidade para controle biológico de $M$. domestica, com a possibilidade de melhores resultados em programas integrados. 
No Brasil, essa espécie já foi descrita parasitando pupas de dípteros em Goiás (Marchiori et al., 2005), Minas Gerais (Marchiori e Silva, 2003), Mato Grosso do Sul (Uchôa-Fernandes et al., 2003) e São Paulo (Costa et al., 2004). Este estudo teve como objetivo relatar a ocorrência de $S$. endius parasitando pupas de $M$. domestica e $S$. calcitrans no sul do Rio Grande do Sul.

As pupas de $M$. domestica e de $S$. calcitrans foram coletadas em fezes de bovinos de leite pelo método da flotação, entre os meses de janeiro e fevereiro de 2008, na Fazenda da Palma/UFPel (3152’00'” S e 52²1'24'” O), município de Capão do Leão, RS. Após a coleta, as pupas foram individualizadas em tubos de ensaio e mantidas em câmara climatizada a $27 \pm 2^{\circ} \mathrm{C}$, com umidade relatiæa $70 \%$ até a emergência das moscas ou dos parasitoides.

A identificação de $S$. endius foi realizada no Instituto Biológico, Agência Paulista de Tecnologia dos Agronegócios (IB-APTA), usando-se a chave proposta por Boucek (1963). O material coletado e identificado foi depositado na Coleção Entomológica do Departamento de Microbiologia e Parasitologia da Universidade Federal de Pelotas.

Foram coletadas 158 pupas de S. calcitrans e 145 pupas de $M$. domestica, das quais emergiram 12 e nove parasitóides, com $7,6 \%$ e $6,2 \%$ de parasitismo, respectivamente. Costa et al. (2004) coletaram pupas de $M$. domestica e $S$. calcitrans em esterco de galinhas poedeiras e encontraram $0,4 \%$ de parasitismo de $S$. endius em pupas de $M$. domestica e 5,6\% em pupas de $S$. calcitrans, totalizando sete e 105 pupas parasitadas, respectivamente.

A ocorrência de $S$. endius parasitando pupas de $M$. domestica e de $S$. calcitrans constitui o primeiro registro de parasitismo dessa espécie para o Rio Grande do Sul e abre a possibilidade de mais uma alternativa para controle biológico de moscas sinantrópicas, em agroecossistemas, viabilizando programas de manejo integrado.

Palavras-chave: mosca doméstica, mosca dos estábulos, inimigo natural

\section{ABSTRACT}

It was reported the occurrence of Spalangia endius Walker, 1839 (Hymenoptera, Pteromalidae) as a parasitoid of pupae of Musca domestica Linnaeus, 1758 (Diptera, Muscidae) and Stomoxys calcitrans Linnaeus, 1758 (Diptera, Muscidae) in the extreme Southern of Brazil. The collection of pupae was performed in January and February, 2008. The pupae of M. domestica and S. calcitrans were collected from bovine feces using the flotation method. The pupae were individualized in glass tubes and maintained in acclimatized chamber at $27 \pm 2{ }^{\circ} \mathrm{C}$ with relative air humidity $\geq 70 \%$ until the emergence of the flies or the parasitoids. The referred occurrence consists in the first report to Rio Grande do Sul.

Keywords: house fly, stable fly, natural enemy

\section{REFERÊNCIAS BIBLIOGRÁFICAS}

BERTI FILHO, E.; THOMAZINI, M.J.; COSTA, V.A. Artrópodes benéficos associados as esterco de galinhas poedeiras no Brasil. Rev. Agric., v.1, p.273-286, 1996.

BITTENCOURT, A.J.; BORJA, G.E.M. Stomoxys calcitrans (Linnaeus, 1758) (Diptera, Muscidae): preferência por locais do corpo de bovinos para alimentação. Rev. Bras. Zoocienc., v.4, p.75-83, 2002

BOUCEK, Z. A taxonomic study in Spalangia Latr. (Hymenoptera: Chalcidoidea). Acta Entomol. Mus. Natl. Pragae, v.35, p.429-512, 1963.
COSTA, V.A.; BERTI FILHO, E.; SILVEIRA NETO, S. Parasitoides (Hymenoptera: Chalcidoidea) de moscas sinantrópicas (Diptera: Muscidae) em aviários de Echaporã, SP. Arq. Inst. Biol., v.71, p.203-209, 2004.

GUIMARÃES, J.H. Moscas dos estábulos, uma importante praga do gado. São Paulo: Agroquímica Ciba Geigy, v.22, p.10-14, 1984.

HANSON, P.E.; GAULD, I.D. The Hymenoptera of Costa Rica. Oxford: Univ. Press, 1995. 893p. 
KAUFMAN, P.E.; LONG, S.J.; RUTZ, D.A. et al. Parasitism rates of Muscidifurax raptorellus and Nasonia vitripennis (Hymenoptera: Pteromalidae) after individual and paired releases en New York poultry facilities. J. Econ. Entomol., v.94, p.593-598, 2001.

MARCHIORI, C.H.; SILVA FILHO, O.M.; BORGES, M.P. et al. Parasitoides da família Pteromalidae (Hymenoptera: Pteromalidae) coletados em fezes de gado bovino em uma propriedade rural do município de Panamá, Goiás. Arq. Bras. Med. Vet. Zootec., p.270-272, 2005.

MARCHIORI, C.H.; SILVA, C.G. First occurrence of parasitoid Spalangia endius (Walker) (Hymenoptera: Pteromalidae) in pupae of Zaprionus indianus Gupta (Diptera: Drosophilidae) in Brazil. Braz. J. Biol., v.63, p.61-362, 2003.
MARICONI, F.A.M.; GUIMARÃES, J.H.; BERTI FILHO, E. A mosca doméstica e algumas outras moscas nocivas. Piracicaba: FEALQ, 1999. $135 \mathrm{p}$.

MORAES, A.P.R.; BADINI, P.V.; DE SOUZA, M.M.S. et al. Avaliação da capacidade de Stomoxys calcitrans (Linnaeus, 1758) em carrear bactérias envolvidas nas etiologias das mastites de municípios do Rio de Janeiro. Rev. Bras. Parasitol. Vet., v.13, p.143-149, 2004.

UCHÔA-FERNANDES, M.A; MOLINA, R.M.S.; OLIVEIRA, O. et al. Larval endoparasitoids (Hymenoptera) of frugivorous flies (Diptera, Tephritoidea) reared from fruits of the cerrado of the State of Mato Grosso do Sul, Brazil. Rev. Bras. Entomol., v.47, p.181-186, 2003. 